Pacific Journal of Mathematics

A MAXIMAL FUNCTION CHARACTERIZATION OF A CLASS 


\title{
A MAXIMAL FUNCTION CHARACTERIZATION OF A CLASS OF HARDY SPACES
}

\author{
ROBYN OWENS
}

\begin{abstract}
In this paper we obtain a maximal function characterisation of a class of Hardy spaces $H^{p}$ which are defined on the upper half plane and combine many of the properties of the classical Hardy spaces for the half plane and the unit disc.
\end{abstract}

Burkholkder, Gundy and Silverstein [1] have shown that the classical Hardy spaces $H^{p}(D)$ and $H^{p}(P)$ on the unit disc $D$ and the upper half plane $P$ can be characterized by the maximal function. For the unit disc they proved the following: if $u$ is an harmonic function in $D$ and $\Omega_{\alpha}(\theta)$ is the Stoltz domain given by the interior of the smallest convex set containing the disc $\{z:|z|<\alpha\}$ and the point $e^{i \theta}$, then $u=\operatorname{Re} F$ for some $F \in H^{p}(D)$ if and only if $\sup \left\{|u(z)|: z \in \Omega_{\alpha}(\theta)\right\} \in L^{p}(\partial D)$, where $\partial D$ denotes the boundary of $D$. This theorem extends an earlier one due to Hardy and Littlewood [9, I, p. 278].

The importance of this result is that a real-valued classification of these spaces of analytic functions allows the notion of Hardy space to be extended to real Euclidean spaces of arbitrary dimension. This was precisely the task undertaken by Fefferman and Stein [2] and in so doing they presented many other equivalent real-valued function characterizations of the corresponding $H^{p}$ spaces.

In the 1950's [5] Hardy spaces were generalized in other directions and different measures on the boundary $\mathbf{R}=\partial P$ were considered. In this paper we will consider a class of Hardy spaces $H^{p}$ which combines many of the properties of the classical Hardy spaces of the upper half plane and the unit disc. Our $H^{p}$ spaces will consist of functions that are analytic in the upper half plane and yet constrained by a bounded measure on its boundary $\mathbf{R}$. This measure arises naturally [5] in the theory of abstract harmonic analysis, where the unit circle is replaced by any abelian locally compact group $G$ and the set of indices over which one forms a "trigonometric series" is taken to be the dual group of $G$. In our case, the group $\mathbf{R}$ of real numbers endowed with the discrete topology is considered; its dual group can then be identified as $b \mathbf{R}$, the Bohr compactification of $\mathbf{R}$ [3]. 
The natural measure which arises by considering the space of generalized analytic functions on $b \mathbf{R}$ [5] turns out to define precisely the condition that the space be an amalgam of $L^{p}$ and $l^{q}$ of $\mathbf{R}[\mathbf{6}]$.

More explicitly, an analytic function $f=u+i \tilde{u}$ belongs to $H^{p}$ if and only if

$$
\sup _{y>0} \sup _{v \in \mathbf{R}} \frac{1}{\pi} \int_{-\infty}^{\infty}|f(x+i y)|^{p} \frac{d x}{1+(v-x)^{2}}<\infty .
$$

When $u$ is harmonic in $P$ its maximal function $u^{*}$ is defined by

$$
u^{*}(t)=\sup \{|u(x+i y)|:|x-t|<y\} .
$$

We shall prove the following three results in connection with characterizing the spaces $H^{p}$ by the maximal function.

(1) If $f=u+i \tilde{u} \in H^{p}, 0<p<\infty$, then

$$
\begin{aligned}
\sup _{v \in \mathbf{R}} \frac{1}{\pi} \int_{-\infty}^{\infty}\left|u^{*}(t)\right|^{p} \frac{d t}{1+(v-t)^{2}} \\
\quad \leq C \sup _{y>0} \sup _{v \in \mathbf{R}} \frac{1}{\pi} \int_{-\infty}^{\infty}|u(x+i y)|^{p} \frac{d x}{1+(v-x)^{2}}
\end{aligned}
$$

where $C_{p}$ is a constant depending only on $p$.

(2) If $u$ is an harmonic function in $P, 0<p<\infty$, and

$$
\sup _{v \in \mathbf{R}} \frac{1}{\pi} \int_{-\infty}^{\infty}\left|u^{*}(t)\right|^{p} \frac{d t}{1+(v-t)^{2}}<\infty
$$

then for every $v^{\prime} \in \mathbf{R}$ there is a conjugate function $\tilde{u}$ of $u$ satisfying

$$
\begin{aligned}
\sup _{y>0} \frac{1}{\pi} \int_{-\infty}^{\infty} & |\tilde{u}(x+i y)|^{p} \frac{d x}{1+\left(v^{\prime}-x\right)^{2}} \\
& \leq C_{p} \sup _{v \in \mathbf{R}} \frac{1}{\pi} \int_{-\infty}^{\infty}\left|u^{*}(t)\right|^{p} \frac{d t}{1+(v-t)^{2}}
\end{aligned}
$$

where $C_{p}$ is a constant depending only on $p$.

(3) If $u$ is harmonic in $P$, if $u^{*}$ satisfies

$$
\sup _{v \in \mathbf{R}} \frac{1}{\pi} \int_{-\infty}^{\infty}\left|u^{*}(t)\right| \frac{d t}{1+(v-t)^{2}}<\infty,
$$

and if $x \mapsto \tilde{u}(x+i y)$ belongs to $L^{\infty}(\mathbf{R})$ for some $y>0$, then

$$
\begin{array}{r}
\sup _{y>0} \sup _{v \in \mathbf{R}} \frac{1}{\pi} \int_{-\infty}^{\infty}|\tilde{u}(x+i y)| \frac{d x}{1+(v-x)^{2}} \\
\leq C \sup _{v \in \mathbf{R}} \frac{1}{\pi} \int_{-\infty}^{\infty}\left|u^{*}(t)\right| \frac{d t}{1+(v-t)^{2}}
\end{array}
$$

and we have $u+i \tilde{u} \in H^{1}$. 
These results give an extension of the Burkholder, Gundy and Silverstein Theorem to our class of Hardy spaces.

2. Notation and preliminary results. We denote by $P$ the open upper half plane and by $\bar{P}$ its closure. Unless otherwise indicated, $L^{p}$ spaces on subsets of the complex plane are taken with respect to Lebesgue measure on the appropriate subset. A mapping $T$ from $L^{1}$ into the set of measurable functions is said to be of weak type $(1,1)$ if

$$
\lambda(\{x:|T f(x)|>\alpha\}) \leq A \alpha^{-1}\|f\|_{1}, \quad f \in L^{1},
$$

where $A$ is a constant independent of $f$ and $\alpha>0$, and $\lambda(E)$ indicates the Lebesgue measure of a set $E$. In this case we say $T f \in L^{1}$-weak. The space $L_{\text {loc }}^{p}$ will consist of those functions $f$ for which $|f|^{p}$ is integrable over every compact subset.

Suppose $0<p<\infty$ and consider the collection of all pairs $(u, \tilde{u})$ where $u$ is an harmonic function in $P, \tilde{u}$ is a conjugate harmonic function of $u$ and both the following are satisfied:

$$
\|u\|_{p}^{p}=\sup _{y>0} \sup _{v \in \mathbf{R}} \frac{1}{\pi} \int_{-\infty}^{\infty}|u(x+i y)|^{p} \frac{d x}{1+(v-x)^{2}}<\infty,
$$

and

$$
\|\tilde{u}\|_{p}^{p}=\sup _{y>0} \sup _{v \in \mathbf{R}} \frac{1}{\pi} \int_{-\infty}^{\infty}|\tilde{u}(x+i y)|^{p} \frac{d x}{1+(v-x)^{2}}<\infty .
$$

If $c$ is a constant and $\tilde{u}$ is a conjugate function of $u$ then so is $\tilde{u}+c$. Moreover, if $\tilde{u}$ satisfies (2.2) then so does $\tilde{u}+c$ since the measure $d x / \pi\left(1+(v-x)^{2}\right)$ has total variation equal to 1 . We define the Hardy space $H^{p}$ as the space of equivalence classes, modulo constants, of all pairs $(u, \tilde{u}+c)$ satisfying (2.1) and (2.2).

If $(u, \tilde{u}) \in H^{p}$ then $f=u+i \tilde{u}$ is an analytic function in $P$ and we say $f \in H^{p}$. When $p \geq 1$ we define the norm of $f$ to be

$$
\|f\|_{p}^{p}=\sup _{y>0} \sup _{v \in \mathbf{R}} \frac{1}{\pi} \int_{-\infty}^{\infty}|f(x+i y)|^{p} \frac{d x}{1+(v-x)^{2}} ;
$$

endowed with this norm, the class of functions $H^{p}$ is a Banach space.

These Hardy spaces have been well-studied and it is known that they display many of the characteristics of the classical Hardy spaces on the disc and the upper half plane. In particular, it is shown in [5] that if $f \in H^{p}, 1 \leq p<\infty$, then the boundary function

$$
f(x)=\lim _{y \rightarrow 0^{+}} f(x+i y)
$$


exists almost everywhere and $f(x+i y)$ can be reproduced as the Poisson integral of $f(x)$. The boundary function $f$ satisfies

$$
\sup _{v \in \mathbf{R}} \int_{-\infty}^{\infty}|f(x)|^{p} \frac{d x}{1+(v-x)^{2}}<\infty
$$

a fact which can also be seen directly using Fatou's Lemma. Denoting by $f_{v}$ the translated and reflected function

$$
f_{v}(x)=f(v-x), \quad x, v \in \mathbf{R},
$$

we have

(2.5) $\sup _{v \in \mathbf{R}} \int_{-\infty}^{\infty}|f(x)|^{p} \frac{d x}{1+(v-x)^{2}}=\sup _{v \in \mathbf{R}} \int_{-\infty}^{\infty}\left|f_{v}(x)\right|^{p} \frac{d x}{1+x^{2}}$.

Let $P_{y}(t)=y / \pi\left(y^{2}+t^{2}\right), y, t \in \mathbf{R}$, denote the Poisson kernel and let $\chi_{I}$ denote the characteristic function of a set $I \subseteq \mathbf{R}$. It is shown in [4] that there exist constants $C_{1}$ and $C_{2}$ such that

$$
\begin{aligned}
C_{1} \sup _{v \in \mathbf{R}} \int_{v}^{v+1}|f(x)|^{p} d x & \leq \sup _{v \in \mathbf{R}} \int_{-\infty}^{\infty}|f(x)|^{p} \frac{d x}{1+(v-x)^{2}} \\
& \leq C_{2} \sup _{v \in \mathbf{R}} \int_{v}^{v+1}|f(x)|^{p} d x,
\end{aligned}
$$

so that by replacing $P_{1}(v-t)$ with the box kernel $\chi_{[v, v+1]}$, the condition (2.4) becomes equivalent to the condition

$$
\sup _{v \in \mathbf{R}} \int_{v}^{v+1}|f(x)|^{p} d x<\infty .
$$

Condition (2.6) is usually expressed [6] by saying that $f$ belongs to the amalgam space $\left(L^{p}, l^{\infty}\right)$, with the quantity on the left hand side of (2.6) denoted by $\|f\|_{p, \infty}^{p}$.

When $u(x+i y)$ is an harmonic function in $P$ satisfying (2.1) and $p \geq 1$, a conjugate function $\tilde{u}$ can be computed explicitly by defining the Hilbert transform on the boundary function $u(x)$. To do this we use the conformal mapping $\psi$ between the unit disc $D$ and the upper half plane given by

$$
\psi(z)=-i\left(\frac{z-1}{z+1}\right), \quad z \in \bar{D}, z \neq-1 .
$$

Via this mapping we can define the Hilbert transform $\mathscr{H} u_{v}$ of each translated function $u_{v}(x)=u(v-x)$, since each $u_{v}$ belonging to $L^{p}(\mathbf{R}$; $\left.d x /\left(1+x^{2}\right)\right)$ is mapped onto a function $u_{v} \circ \psi$ belonging to $L^{p}(\mathbf{T})$ and the usual Hilbert transform $H$ is well-defined on this space $(\mathbf{T}=\partial D$ 
denotes the unit circle). Explicitly, $\mathscr{H} u_{v}$ is defined as $H\left(u_{v} \circ \psi\right) \circ \psi^{-1}$. Under the conformal mapping $\psi$ however, the translations of $u$ are not preserved; that is, in general $\mathscr{H} u_{v} \neq(\mathscr{H} u)_{v}$. But since (the Poisson integrals of) $\mathscr{H} u_{v}$ and $(\mathscr{H} u)_{v}$ both represent the imaginary part of the same analytic function their difference can only be a constant; we write $\mathscr{H} u_{v}-(\mathscr{H} u)_{v}=c(u, v)$.

A theorem of M. Riesz implies that $\mathscr{H} u_{v} \in L^{p}\left(\mathbf{R} ; d x /\left(1+x^{2}\right)\right)$ whenever $u_{v} \in L^{p}\left(\mathbf{R} ; d x /\left(1+x^{2}\right)\right)$, which entails that for $1<p<\infty$

$$
\begin{aligned}
\int_{-\infty}^{\infty}\left|\mathscr{H} u_{v}(x)\right|^{p} \frac{d x}{1+x^{2}} & \leq C_{p} \cdot \int_{-\infty}^{\infty}\left|u_{v}(x)\right|^{p} \frac{d x}{1+x^{2}} \\
& \leq C_{p} \sup _{v^{\prime} \in \mathbf{R}} \int_{-\infty}^{\infty}\left|u_{v^{\prime}}(x)\right|^{p} \frac{d x}{1+x^{2}}
\end{aligned}
$$

for some constant $C_{p}$ which depends only on $p$. In other words we have

THEOREM 1. If $u$ is an harmonic function in $P$ satisfying (2.1) and $1<p<\infty$ then for every $v \in \mathbf{R}$ there is a conjugate function $\tilde{u}$ of $u$ such that

$$
\int_{-\infty}^{\infty}|\tilde{u}(x)|^{p} \frac{d x}{1+(v-x)^{2}} \leq C_{p} \cdot\|u\|_{p, \infty}^{p}
$$

where $C_{p}$ is a constant depending only on $p$.

3. The Main Theorem: Direct half and converse for $p>1$. In view of the Burkholder, Gundy and Silverstein Theorem [1, p. 138] it is natural to ask whether the spaces $H^{p}, 0<p<\infty$, can be characterized by the non-tangential maximal function. For each $t \in \mathbf{R}$ we denote by $\Gamma(t)$ the cone

$$
\Gamma(t)=\{z=x+i y \in P:|x-t|<y\},
$$

and if $u$ is an harmonic function on $P$ we define the non-tangential maximal function $u^{*}$ of $u$ by

$$
u^{*}(t)=\sup _{z \in \Gamma(t)}|u(z)|, \quad t \in \mathbf{R} .
$$

In this section we shall prove the following two results.

TheOREM 2. If $(u, \tilde{u}) \in H^{p}$ and $0<p<\infty$ then $u^{*} \in\left(L^{p}, l^{\infty}\right)$.

THEOREM 3. If $u$ is an harmonic function in $P$ and $u^{*} \in\left(L^{p}, l^{\infty}\right)$, $1<p<\infty$, then for every $v \in \mathbf{R}$ there is a conjugate function $\tilde{u}$ that 
satisfles

$$
\int_{-\infty}^{\infty}|\tilde{u}(x)|^{p} \frac{d x}{1+(v-x)^{2}} \leq C_{p}\|u\|_{p}^{p}
$$

where $C_{p}$ is a constant depending only on $p$.

In Theorem 3 the hypothesis that $u^{*} \in\left(L^{p}, l^{\infty}\right)$ certainly implies that $u$ satisfies (2.1) since $u^{*}$ dominates $u$ on $\mathbf{R}$ and the Poisson integral is norm-decreasing [4]. Nevertheless, the Theorem does not state that $(u, \tilde{u})$ $\in H^{p}$ since $\tilde{u}$ does not necessarily satisfy (2.2). In $\S 6$ we will discuss conditions under which (2.2) is satisfied.

To prove Theorem 2 we begin with a lemma.

LeMma 1. If $F$ is analytic in $P$ and

$$
\|F\|_{p}^{p}=\sup _{y>0} \sup _{v \in \mathbf{R}} \int_{-\infty}^{\infty}|F(x+i y)|^{p} \frac{d x}{1+(v-x)^{2}}<\infty,
$$

then for every $\varepsilon>0,|F|^{p}$ is bounded on

$$
\bar{P}_{\varepsilon}=\{x+i y \in P: y \geq \varepsilon\} .
$$

Proof. For $p \geq 1$ this is a trivial consequence of the fact that $F$ can be expressed as the Poisson integral of its boundary values.

When $p<1$ we note that $|F|^{p}$ is subharmonic whenever $F$ is analytic. Given $x+i y \in P$ we choose $h<1$ small enough so that the disc $\{\xi+i \eta$ : $\left.(x-\xi)^{2}+(y-\eta)^{2} \leq h\right\} \subseteq P$. Then

$$
\begin{aligned}
|F(x+i y)|^{p} & \leq \frac{C}{h^{2}} \underset{(x-\xi)^{2}+(y-\eta)^{2} \leq h}{\iint_{y-h}|F(\xi+i \eta)|^{p} d \xi d \eta} \\
& =\frac{C}{h^{2}} \iint|F(\xi+i \eta)|^{p} \frac{1+(x-\xi)^{2}}{1+(x-\xi)^{2}} d \xi d \eta \\
& \leq \frac{C}{h^{2}}(1+h) \int_{y-h}^{y+h}\|F\|_{p}^{p} d \eta \leq \frac{C}{h^{2}}(1+h) 2 h\|F\|_{p}^{p} .
\end{aligned}
$$

Proof of Theorem 2. Consider the conformal mapping $\psi$ defined by (2.7). Under the inverse of $\psi$, cones

$$
\Gamma_{\alpha}(t)=\{x+i y \in P:|x-t| \leq \alpha y\}
$$



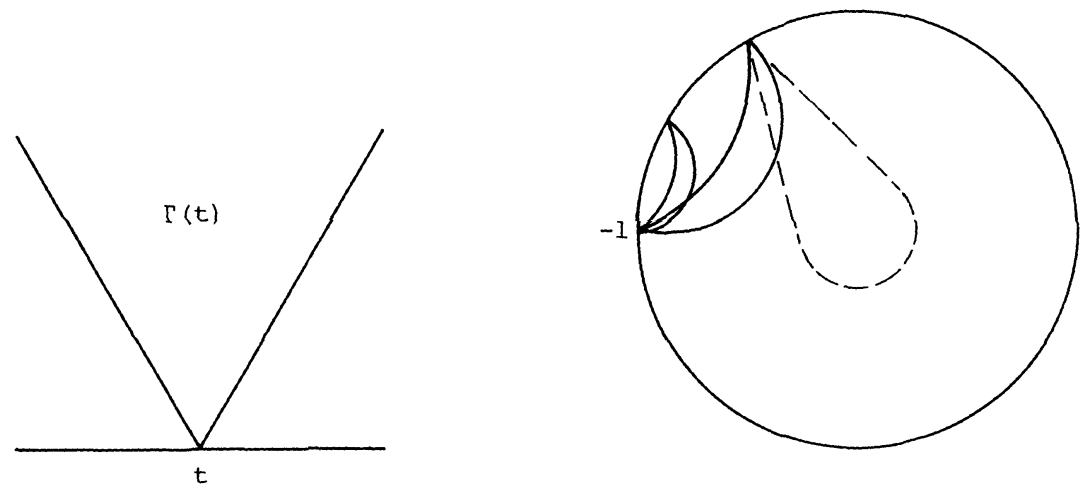

Figure 1
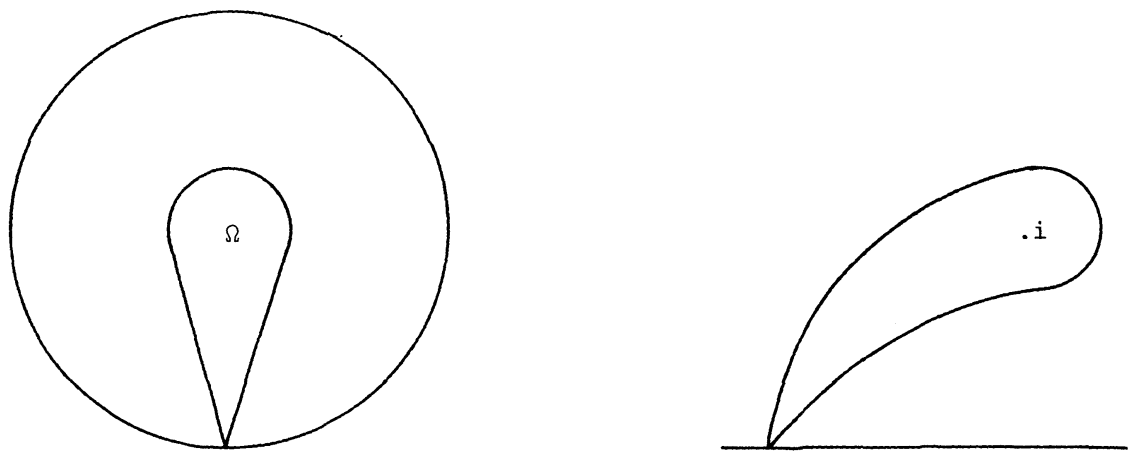

FIGURE 2

are mapped onto moon-shaped regions in the disc and as $t \rightarrow \pm \infty$ these regions become smaller. See Figure 1. In the other direction, a Stoltz domain $\Omega_{\alpha}(\theta)$ in the disc, defined as the interior of the smallest convex set containing the disc $\{z:|z|<\alpha\}$ and the point $e^{i \theta}$, is mapped by $\psi$ onto a bounded region in the upper half plane containing the point $i$. See Figure 2. Since $\psi$ is conformal, the angle at the apex is always preserved.

Also under $\psi$, the pair $(u, \tilde{u}) \in H^{p}$ is transformed into a pair of functions $u \circ \psi$ and $\tilde{u} \circ \psi$ satisfying

$$
\begin{aligned}
& \|u \circ \psi\|_{p}^{p}=\sup _{r<1} \frac{1}{2 \pi} \int_{0}^{2 \pi}\left|u \circ \psi\left(r e^{i \theta}\right)\right|^{p} d \theta<\infty, \\
& \|\tilde{u} \circ \psi\|_{p}^{p}=\sup _{r<1} \frac{1}{2 \pi} \int_{0}^{2 \pi}\left|\tilde{u} \circ \psi\left(r e^{i \theta}\right)\right|^{p} d \theta<\infty .
\end{aligned}
$$


These relations assert that $(u \circ \psi, \tilde{u} \circ \psi) \in H^{p}(D)$, the classical Hardy space on the unit disc. As $(u \circ \psi)$ is also a conjugate function of $u \circ \psi$ this means $(u \circ \psi,(u \circ \psi) \tilde{)}) \in H^{p}(D)$ and by the Hardy-Littlewood Maximal Theorem [9, Theorem 7.36] we have $(u \circ \psi)^{*} \in L^{p}(\mathbf{T})$ with $\left\|(u \circ \psi)^{*}\right\|_{p}^{p} \leq$ $C_{p}\|u \circ \psi\|_{p}^{p}$.

By Lemma 2 however, $(u \circ \psi) *$ can be calculated by considering $|u \circ \psi(z)|$ for $z$ belonging to a truncated Stoltz domain; so that outside an $\varepsilon$-neighbourhood of the point -1 , the behaviour of $u^{*} \circ \psi$ (which can be determined by considering $|u \circ \psi(z)|$ over truncated moons) is controlled by that of $(u \circ \psi)^{*}$ and we have $u^{*} \circ \psi \in L_{\text {loc }}^{p}(\mathbf{T} \backslash\{-1\})$. Returning to the upper half plane, we see that $u^{*} \in L_{p_{x}}(\mathbf{R})$.

This implies that for each $v \in \mathbf{R}$ we have

$$
\int_{v}^{v+1}\left|u^{*}(t)\right|^{p} d t \leq K_{p, \varepsilon}\|u\|_{p}^{p}
$$

where $K_{p, \varepsilon}$ is a constant which depends on $p$ and $\varepsilon$ but is independent of $v \in \mathbf{R}$, since $\left(u^{*}\right)_{v}=\left(u_{v}\right)^{*}$. Thus

$$
\sup _{v \in \mathbf{R}} \int_{v}^{v+1}\left|u^{*}(t)\right|^{p} d t \leq K\|u\|_{p}^{p}
$$

and we have proved that $u^{*} \in\left(L^{p}, l^{\infty}\right)$.

Proof of Theorem 3. Suppose $p>1, u$ is an harmonic function in $P$ and $u^{*} \in\left(L^{p}, l^{\infty}\right)$. A trivial consequence of Theorem 1 is that for each $v \in \mathbf{R}$ there is a conjugate function $\tilde{u}$ of $u$ such that

$$
\int_{-\infty}^{\infty}|\tilde{u}(x)|^{p} \frac{d x}{1+(v-x)^{2}} \leq C_{p}\|u\|_{p, \infty}^{p} \leq C_{p}\left\|u^{*}\right\|_{p, \infty}^{p},
$$

where $C_{p}$ is a constant depending only on $p$.

4. The converse: $p=1$. To treat the case $p=1$ we will use certain properties of conformal mappings. Consider a disc $\Delta$ in the plane $\mathbf{C}$ the circumference of which passes through the points 0 and 1 and let $\Omega$ denote that portion of $\Delta$ lying above the real axis. The region $\Omega$ can be mapped conformally onto $D$ by using the function $F=f_{3} \circ f_{2} \circ f_{1}$, where $f_{1}$ and $f_{3}$ are linear fractional transformations and $f_{2}$ is a power; more explicitly, we put

$$
\begin{aligned}
& f_{1}(z)=-z(z-1) \\
& f_{2}(z)=z^{\pi / \alpha} \text { and } \\
& f_{3}(z)=(z-i) /(z+i)
\end{aligned}
$$

where $\alpha$ denotes the angle formed by $f_{1}(\partial \Omega)$. 
One can easily check that $F^{-1}$ transforms radii of $D$ into path segments emanating from one point inside $\Omega$ and terminating on $\partial \Omega$. Moreover, the mapping $F^{-1}$ guarantees that each segment will be contained in some Stoltz domain in $\Delta$ and each Stoltz domain can be chosen to have the same opening angle. The $L^{p}$ classes are also preserved under $F$ in the sense that Lebesgue measure on the circle is transformed under $F^{-1}$ to a measure on $\partial \Omega$ which is absolutely continuous with respect to Lebesgue measure.

THeOREM 4. If $u$ is harmonic in $P$ and $u^{*} \in\left(L^{1}, l^{\infty}\right)$ then for every $v \in \mathbf{R}$ there is a conjugate function $\tilde{u}$ of $u$ such that

$$
\int_{-\infty}^{\infty}|\tilde{u}(x)| \frac{d x}{1+(v-x)^{2}} \leq C\left\|u^{*}\right\|_{1, \infty}
$$

where $C$ is some universal constant.

To prove this theorem we shall use the following result which appears in $[2$, p. 170]. When $u$ is an harmonic function in $D$ its radial maximal function $u^{+}$is defined by

$$
u^{+}(\theta)=\sup _{r<1}\left|u\left(r e^{i \theta}\right)\right|, \quad \theta \in \mathbf{T} .
$$

Fefferman and Stein showed that $u$ is the real part of some analytic $f \in H^{p}(D), 0<p<\infty$, if and only if $u^{+} \in L^{p}(\mathbf{T})$.

The proof of Theorem 4 is subdivided into a number of lemmas. As before, to define the Hilbert transform we use the conformal mapping $\psi$ defined in (2.7). Putting $v=u \circ \psi$ we see that the hypotheses of the theorem imply that the corresponding maximal function, which we now denote by $v^{*}$, belongs to $L_{\text {loc }}^{1}(\mathbf{T} \backslash\{-1\})$. Consequently, for the disc we have $v \in L^{1}(\mathbf{T})$ and $v^{*} \in L_{\mathrm{loc}}^{1}(\mathbf{T} \backslash\{-1\})$. By the Kolmogorov Theorem $\left[8\right.$, p. 187] we know that $\tilde{v}$ is well-defined and in $L_{\text {weak }}^{1}(T)$.

LEMMA 2. Under the hypotheses of Theorem 4,

$$
\tilde{v} \in L_{\mathrm{loc}}^{1}(\mathbf{T} \backslash\{-1\}) .
$$

Proof. Consider the region $\Sigma$ defined as $D \backslash H$ where $H$ is a small rectangular region centred about the point -1 with sides parallel to the $x$ and $y$ axes, one side forming a chord of $D$. Let $F: \Sigma \rightarrow D$ be a conformal mapping between $\Sigma$ and the unit disc, so that $v \circ F^{-1}$ is harmonic in $D$. 
We can dominate the radial maximal function $\left(v \circ F^{-1}\right)^{+}$by the non-tangential maximal function $v^{*}$ since under $F^{-1}$ there is a one to one correspondence between each radius $R(\theta)=\left\{r e^{i \theta}: 0 \leq r<1\right\}, \theta \in \mathbf{T}$, and a corresponding Stoltz domain $\Omega\left(\theta^{\prime}\right), \theta^{\prime} \in \mathbf{T}$. More explicitly,

$$
\begin{aligned}
\left(v \circ F^{-1}\right)^{+}(\theta) & =\sup _{r<1}\left|f\left(F^{-1}\left(r e^{i \theta}\right)\right)\right| \\
& \leq \sup _{r e^{i t} \in \Omega\left(\theta^{\prime}\right)}\left|v\left(r e^{i t}\right)\right|=v^{*}\left(\theta^{\prime}\right) .
\end{aligned}
$$

Whenever $N_{\varepsilon}(-1)$ denotes an $\varepsilon$-neighbourhood of -1 contained strictly inside the rectangle $H$ we have $\left(v \circ F^{-1}\right)^{+}(\theta) \leq C$ uniformly in $\theta \in \mathbf{T} \backslash N_{\varepsilon}(-1)$ since, for each $\theta,\left(v \circ F^{-1}\right)^{+}(\theta)$ is calculated by taking the supremum of $|v|$ over some compact subset of $D$. Since $v^{*} \in$ $L_{\text {loc }}^{1}(\mathbf{T} \backslash\{-1\})$ and $\left(v \circ F^{-1}\right)^{+} \leq v^{*}$ we see that $\left(v \circ F^{-1}\right)^{+}$is locally integrable on $\mathbf{T} \backslash\{-1\}$ and uniformly bounded in a neighbourhood of -1. Consequently $\left(v \circ F^{-1}\right)^{+} \in L^{1}(\mathbf{T})$.

By the theorem of Fefferman and Stein [2, p. 17], $\left(v \circ F^{-1}\right)^{\sim} \in L^{1}(\mathbf{T})$ and since $\tilde{v}=\left(v \circ F^{-1}\right)^{\tilde{}} \circ F$ (modulo constants) we conclude that $\tilde{v} \in$ $L_{\text {loc }}^{1}(\mathbf{T} \backslash\{-1\})$. This completes the proof.

Interpreting Lemma 2 in the upper half plane we have the following situation: both $u$ and $u^{*}$ belong to $\left(L^{1}, L^{\infty}\right)$ and any conjugate $\tilde{u}$ of $u$ is in $L_{\text {loc }}^{1}(\mathbf{R})$. In particular, for every $v \in \mathbf{R}$ there is a conjugate function $\tilde{u}$ of $u$ satisfying

$$
\int_{v}^{v+1}|\tilde{u}(x)| d x \leq K\left\|u^{*}\right\|_{1, \infty}
$$

where $K$ is a constant independent of $u$. Necessarily, $K$ is also independent of $v \in \mathbf{R}$.

LEMMA 3. With the same hypotheses as Theorem 4, condition (4.1) implies

$$
\frac{1}{|I|} \int_{I}|\tilde{u}(x)| d x \leq K\left\|u^{*}\right\|_{1, \infty}
$$

where $I$ is any interval of integral length and $K$ is a universal constant.

Proof. To determine how the constant $K$ in (4.1) changes when the interval is expanded we suppose without loss of generality that $I=(0, n)$ and define $u^{\#}(x)=u(n x)$. Then

$$
K\left\|\left(u^{\#}\right)^{*}\right\|_{1, \infty} \geq \int_{0}^{1}\left|\tilde{u}^{\#}(x)\right| d x=\int_{0}^{1}|\tilde{u}(n x)| d x=\frac{1}{n} \int_{0}^{n}|\tilde{u}(x)| d x .
$$


But

$$
\begin{aligned}
\left\|\left(u^{\#}\right)^{*}\right\|_{1, \infty} & =\sup _{v \in \mathbf{R}} \int_{v}^{v+1}\left|u^{*}(n t)\right| d t=\sup _{v \in \mathbf{R}} \int_{n v}^{n v+n}\left|u^{*}(x)\right| \frac{d x}{n} \\
& =\frac{1}{n} \sup _{v \in \mathbf{R}}\left[\int_{n v}^{n v+1}+\int_{n v+1}^{n v+2}+\cdots+\int_{n v+n-1}^{n v+n}\left|u^{*}(x)\right| d x\right] \\
& \leq\left\|u^{*}\right\|_{1, \infty} .
\end{aligned}
$$

Thus

$$
\frac{1}{n} \int_{0}^{n}|\tilde{u}(x)| d x \leq K\left\|u^{*}\right\|_{1, \infty}
$$

and the proof is complete. Note that we now have a BMO-type condition [4] on $\tilde{u}$ for large intervals.

LemMa 4. Let $n \in \mathbf{N}$ and denote by $\tilde{u}_{n}$ a conjugate function of $u$ that satisfies (4.2) for the interval $\left[2^{n}, 2^{n+1}\right]$. Then

$$
\left|\tilde{u}_{n}-\tilde{u}_{n-1}\right| \leq C\left\|u^{*}\right\|_{1, \infty}
$$

where $C$ is a constant that does not depend on $n$.

Proof. Note first of all that $\tilde{u}_{n}$ and $\tilde{u}_{n-1}$ are both conjugate functions of $u$ so their difference can only be a constant. To begin, we consider the effect of doubling the interval. Let $I_{1}, I_{2}$ and $I_{3}$ be real intervals of the form $I_{1}=(z,(a+b) / 2), I_{2}=((a+b) / 2, b)$ and $I_{3}=(a, b)$ and let $\tilde{u}_{1}$, $\tilde{u}_{2}$ and $\tilde{u}_{3}$ be conjugates on $\tilde{u}$ which satisfy (4.2) for the intervals $I_{1}, I_{2}$ and $I_{3}$ respectively. We have

$$
\begin{aligned}
K\left\|u^{*}\right\|_{1, \infty} & \geq \frac{1}{\left|I_{3}\right|} \int_{I_{3}}\left|\tilde{u}_{3}(x)\right| d x \\
& =\frac{1}{\left|I_{3}\right|}\left\{\int_{I_{1}}\left|\tilde{u}_{3}(x)\right| d x+\int_{I_{2}}\left|\tilde{u}_{3}(x)\right| d x\right\} .
\end{aligned}
$$

but also

$$
K\left\|u^{*}\right\|_{1, \infty} \geq \frac{1}{2\left|I_{1}\right|} \int_{I_{1}}\left|\tilde{u}_{1}(x)\right| d x+\frac{1}{2\left|I_{2}\right|} \int_{I_{2}}\left|\tilde{u}_{2}(x)\right| d x .
$$

Since each integral is positive (4.3) gives us

$$
\frac{1}{\left|I_{3}\right|} \int_{I_{1}}\left|\tilde{u}_{3}(x)\right| d x \leq K\left\|u^{*}\right\|_{1, \infty}
$$


whilst (4.4) gives

$$
\frac{1}{\left|I_{3}\right|} \int_{I_{1}}\left|\tilde{u}_{1}(x)\right| d x \leq K\left\|u^{*}\right\|_{1, \infty}
$$

so that

$$
\frac{1}{2}\left|\tilde{u}_{3}-\tilde{u}_{1}\right|=\frac{1}{\left|I_{3}\right|} \int_{I_{1}}\left|\tilde{u}_{3}(x)-\tilde{u}_{1}(x)\right| d x \leq 2 K\left\|u^{*}\right\|_{1, \infty} .
$$

In a similar way we find

$$
\frac{1}{2}\left|\tilde{u}_{3}-\tilde{u}_{2}\right| \leq 2 K\left\|u^{*}\right\|_{1, \infty} .
$$

As in the hypotheses we now let $\tilde{u}_{n}$ be a conjugate of $u$ associated with the interval $\left[2^{n}, 2^{n+1}\right]$ in (4.2) and let $\tilde{\mu}_{n}$ be a conjugate of $u$ associated with the interval $\left[0,2^{n}\right]$. Using the reasoning employed above we have

$$
\left|\tilde{\mu}_{n}-\tilde{u}_{n}\right| \leq\left|\tilde{\mu}_{n}-\tilde{\mu}_{n+1}\right|+\left|\tilde{\mu}_{n+1}-\tilde{u}_{n}\right| \leq 8 K\left\|u^{*}\right\|_{1, \infty}
$$

and

$$
\left|\tilde{\mu}_{n}-\tilde{u}_{n-1}\right| \leq 4 K\left\|u^{*}\right\|_{1, \infty}
$$

so that finally

$$
\left|\tilde{u}_{n}-\tilde{u}_{n-1}\right| \leq 12 K\left\|u^{*}\right\|_{1, \infty}
$$

and the proof is complete. Note that this means that as the interval is moved from $[0,1]$ to $\left[2^{n}, 2^{n+1}\right]$ a conjugate function $\tilde{u}$ of $u$ is changing at most by a constant which is $O(n)$.

Finally, to fix a conjugate once and for all we denote by $\tilde{u}$ a conjugate of $u$ associated with the interval $(0,1)$, as in (4.2). Using the estimates we have made in Lemmas 3 and 4 we see that

$$
\int_{2^{n}}^{2^{n+1}}|\tilde{u}(x)| \frac{d x}{1+x^{2}} \leq \frac{2^{n} C n+2^{n} K\left\|u^{*}\right\|_{1, \infty}}{2^{2 n}}
$$

where $C$ and $K$ are constants. This shows that $\tilde{u} \in L^{1}\left(\mathbf{R} ; d x /\left(1+x^{2}\right)\right)$. But there is nothing special about the interval $(0,1)$ and the same argument applies with equal validity to any interval $(v, v+1), v \in \mathbf{R}$.

In conclusion, we have shown that there is a constant $C$ such that if $u$ is harmonic in $P$ and $u^{*} \in\left(L^{1}, l^{\infty}\right)$ then for every $v \in \mathbf{R}$ there exists a conjugate function $\tilde{u}$ of $u$ satisfying

$$
\int_{-\infty}^{\infty}|\tilde{u}(x)| \frac{d x}{1+(v-x)^{2}} \leq C\left\|u^{*}\right\|_{1, \infty} .
$$


5. The converse: $p<1$. When $p<1$ we can no longer represent the harmonic function $u$ as a Poisson integral of some function on the boundary. Nevertheless, the proof we gave in the case $p=1$ can be adapted to this situation and the key idea is to use the maximal conjugate function. When $f$ is harmonic in $D$ and $\tilde{f}$ is a conjugate of $f$ the maximal conjugate function $\tilde{f}^{*}$ is defined by

$$
(\tilde{f})^{*}(\boldsymbol{\theta})=\sup _{z \in \Omega(\theta)}|\tilde{f}(z)|, \quad \boldsymbol{\theta} \in \mathbf{T} .
$$

Fefferman and Stein showed that if $f$ is an harmonic function such that $f^{*} \in L^{p}(\mathbf{T}), 0<p<\infty$, then there is a conjugate function $\tilde{f}$ such that $\tilde{f}^{*} \in L^{p}(\mathbf{T})$ and

$$
\left\|\tilde{f}^{*}\right\|_{p} \leq C_{p}\left\|f^{*}\right\|_{p}
$$

where $C_{p}$ is a constant depending only on $p[4$, Ch. 3].

THeOREM 5. If $u$ is harmonic in $P$ and $u^{*} \in\left(L^{p}, l^{\infty}\right), 0<p<1$, then for every $v \in \mathbf{R}$ there is a conjugate function $\tilde{u}$ of $u$ such that

$$
\sup _{y>0} \int_{-\infty}^{\infty}|\tilde{u}(x+i y)|^{p} \frac{d x}{1+(v-x)^{2}} \leq C_{p}\left\|u^{*}\right\|_{p, \infty}^{p} .
$$

The proof of this theorem follows the same lines as that of Theorem 4 and so we restrict ourselves to an outline, only giving details when differences occur.

Via the conformal mapping $\psi$ we transfer to the unit disc and put $v=u \circ \psi$. Then $v$ is harmonic in $D$ and $v^{*} \in L_{\text {loc }}^{p}(\mathbf{T} \backslash\{-1\})$. We will denote by $\tilde{v}$ a conjugate function of $v$ and by $v_{r}$ the function $v$ restricted to the circle $r \mathbf{T}=\left\{r e^{i \theta}: 0 \leq \theta<2 \pi\right\}$ where $r<1$.

Lemma 5. $\tilde{v}^{+} \in L_{\text {loc }}^{p}(\mathbf{T} \backslash\{-1\})$.

Proof. Arguing as in Lemma 2 we consider the region $\Sigma=D \backslash H$ where $H$ is once again a small rectangular region centred about the point -1 and let $F: \Sigma \rightarrow D$ be a conformal mapping between $\Sigma$ and the unit disc. From a theorem of Fefferman and Stein we deduce that $v \circ F^{-1}$ is the real part of some analytic function in $H^{p}(D)$ and so it follows from the Burkholder, Gundy and Silverstein theorem that $\left(v \circ F^{-1}\right)^{*} \in L^{p}(\mathbf{T})$. This implies that $\left(\widetilde{v \circ F^{-1}}\right) * L^{p}(\mathbf{T})$. 
Recalling that $\tilde{v}=\left(v \circ F^{-1}\right) \tilde{}{ }^{\tilde{T}} \circ F$ (modulo constants) we now calculate $\tilde{v}^{+}(\theta)$ for those $\theta \in \partial \Sigma \cap \mathbf{T}$. As before, a radial segment in $\Sigma$ is transformed under $F$ into a path segment in $D$ contained in some Stoltz domain. So

$$
\begin{aligned}
\tilde{v}^{+}(\theta) & =\sup _{r<1}\left|\left(r e^{i \theta}\right)\right| \\
& =\sup _{r<1}\left|\left(v \circ F^{-1}\right)^{\sim}\left(F\left(r e^{i \theta}\right)\right)\right| \quad \text { (modulo constants) } \\
& \leq\left(\widetilde{v \circ F^{-1}}\right)^{*}(F(\theta))
\end{aligned}
$$

and we conclude that $\tilde{v}^{+} \in L_{\text {loc }}^{p}(\mathbf{T} \backslash\{-1\})$.

Transferring this back to the upper half plane as before we have for every $v \in \mathbf{R}$ the existence of a conjugate function $\tilde{u}$ satisfying

$$
\sup _{y>0} \int_{v}^{v+1}|\tilde{u}(x+i y)|^{p} d x \leq C_{p}\left\|u^{*}\right\|_{p, \infty}^{p}
$$

where $C$ is a constant depending on $p$.

In the same fashion as Lemma 3 this can be extended to

$$
\sup _{y>0} \frac{1}{|I|} \int_{I}|\tilde{u}(x+i y)|^{p} d x \leq C_{p}\left\|u^{*}\right\|_{p, \infty}^{p}
$$

where $I$ is any interval of integral length. The conjugate function $\tilde{u}$ depends on the interval $I$ in the sense described in Lemma 4, the proof being identical but for the replacement of the triangle inequality by the inequality $|a+b|^{p} \leq|a|^{p}+|b|^{p}$ which is valid for all $p<1$. This completes the outline of the proof of Theorem 5 .

6. A direct classification. We have shown that if $u$ is harmonic in $P$ and $u=\operatorname{Re} f$ for some $f \in H^{p}, 0<p<\infty$, then $u^{*} \in\left(L^{p}, l^{\infty}\right)$; on the other hand, the condition that $u^{*} \in\left(L^{p}, l^{\infty}\right)$ certainly implies $u \in$ $\left(L^{p}, l^{\infty}\right)$ and consequently that the harmonic extension $u(x+i y)$ satisfies (2.1), but we can only say that for each $v \in \mathbf{R}$ there is a conjugate function $\tilde{u}$ satisfying

$$
\sup _{y>0} \frac{1}{\pi} \int_{-\infty}^{\infty}|\tilde{u}(x+i y)|^{p} \frac{d x}{1+(v-x)^{2}} \leq C_{p}\left\|u^{*}\right\|_{p, \infty}^{p} .
$$

In this section we shall show that the addition of a boundedness condition implies that $\tilde{u}$ actually satisfies (2.2). In this case we can speak of the conjugate function $\tilde{u}$ and state that $(u, \tilde{u}) \in H^{p}$. We treat the case $p=1$. 
THEOREM 6. Suppose $u$ is harmonic in $P$ and $u^{*} \in\left(L^{1}, l^{\infty}\right)$. If $x \rightarrow$ $\tilde{u}(x+i y)$ belongs to $L^{\infty}(\mathbf{R})$ for some $y>0$ then $\tilde{u} \in\left(L^{1}, l^{\infty}\right)$.

Proof. Choose any $v \in \mathbf{R}$. Using the notation of (4.2) we let $\tilde{u}$ denote a conjugate function of $u$ which satisfies

$$
\int_{0}^{1}|\tilde{u}(x)| d x \leq K\left\|u^{*}\right\|_{1, \infty} .
$$

For any $n \geq 1$ there is a constant $\lambda_{n}(v)$ such that

$$
\frac{1}{2^{n-1}} \int_{v+2^{n-1}}^{v+2^{n}}\left|\tilde{u}(x)-\lambda_{n}(v)\right| d x \leq K\left\|u^{*}\right\|_{1, \infty}
$$

and we know $\lambda_{n}(v)=O(n)$. Let $\lambda_{0}(v)$ denote that constant such that $\tilde{u}-\lambda_{0}$ satisfies (4.2) for the interval $[v-1, v+1]$. Then

$$
\begin{aligned}
& \tilde{u}(v+i y)=\int_{-\infty}^{\infty} P_{y}(v-t) \tilde{u}(t) d t \\
& =\int_{-\infty}^{\infty} P_{y}(v-t)\left[\tilde{u}(t)-\lambda_{0}(v)\right] d t+\lambda_{0}(v) \\
& =\sum_{n \in \mathbf{N}} \int_{v-2^{n+1}}^{v-2^{n}}+\int_{v+2^{n}}^{v+2^{n+1}}+\int_{v-1}^{v+1} P_{y}(v-t)\left[\tilde{u}(t)-\lambda_{0}(v)\right] d t+\lambda_{0}(v) .
\end{aligned}
$$

Now

$$
\begin{aligned}
\left|\tilde{u}-\lambda_{0}(v)\right| & \leq\left|\tilde{u}-\lambda_{n+1}(v)\right|+\left|\lambda_{n+1}(v)-\lambda_{n}(v)\right|+\cdots+\left|\lambda_{1}(v)-\lambda_{0}(v)\right| \\
& \leq\left|\tilde{u}-\lambda_{n+1}(v)\right|+(n+1) C\left\|u^{*}\right\|_{1, \infty}
\end{aligned}
$$

by Lemma 4 , and by (6.1) we have

$$
\int_{v+2^{n}}^{v+2^{n+1}}\left|\tilde{u}(t)-\lambda_{n+1}(v)\right| d t \leq 2^{n} K\left\|u^{*}\right\|_{1, \infty}
$$

so that for any $n \in \mathbf{N}$ we have

$$
\int_{v+2^{n}}^{v+2^{n+1}} P_{y}(v-t)\left|\tilde{u}(t)-\lambda_{0}(v)\right| d t \leq \frac{2^{n} K\left\|u^{*}\right\|_{1, \infty}+(n+1) C\left\|u^{*}\right\|_{1, \infty}}{2^{2 n}}
$$

A similar estimate works over the interval $\left[v-2^{n}, v-2^{n-1}\right]$ so that we deduce

$$
\left|\int_{-\infty}^{\infty} P_{y}(v-t)\left[\tilde{u}(t)-\lambda_{0}(v)\right] d t\right| \leq N<\infty
$$

where $N$ is a constant depending on the constants $K, C$ and $\left\|u^{*}\right\|_{1, \infty}$ but is independent of $v \in \mathbf{R}$. 
Now since $v \rightarrow \tilde{u}(v+i y) \in L^{\infty}(\mathbf{R})$ and

$$
\tilde{u}(v+i y)=\int_{-\infty}^{\infty} P_{y}(v-t)\left[\tilde{u}(t)-\lambda_{0}(v)\right] d t+\lambda_{0}(v),
$$

we see that $\lambda_{0}(v)$ must be bounded for all $v \in \mathbf{R}$. In other words, the constants representing the difference between any two conjugates of $u$ are bounded and hence may be disregarded. This implies $\tilde{u} \in\left(L^{1}, l^{\infty}\right)$.

7. Acknowledgments. Most of this work appears as Chapter 2 in [6], which was admitted as a D. Phil thesis at Oxford University in 1980. I would like to thank N. Th. Varopoulos for all his kind help and encouragement whilst I concluded the work in 1979 at the Universite de Paris-Sud at Orsay. Many others have helped in various ways but it was mostly his persistence and unending supply of good ideas which were responsible for its completion.

\section{REFERENCES}

[1] D. L. Burkholder, R. F. Gundy and M. L. Silverstein, A maximal function characterization of the class $H^{p}$, Trans. Amer. Math. Soc., 157 (1971), 137-153.

[2] C. Fefferman and E. M. Stein, $H^{p}$ Spaces of several variables, Acta. Math., 129 (1972), 137-193.

[3] E. Følner, On the Dual Spaces of the Besicovitch almost periodic Spaces, Dan. Mat. Fys. Medd., 29, No. 1 (1954).

[4] J. Garnett, Bounded Analytic Functions, Academic Press (1980).

[5] K. Hoffman, Boundary behaviour of generalized analytic functions, Trans. Amer. Math. Soc., 87 (1958), 447-466.

[6] F. Holland, Harmonic analysis on amalgams of $L^{p}$ and $l^{q}$, J. London Math. Soc., (2), 10 (1975), 295-305.

[7] R. Owens, Almost Periodic Hardy Spaces, D. Phil. Thesis, Oxford University, (1980).

[8] E. M. Stein and G. Weiss, Introduction to Fourier Analysis on Euclidean Spaces, Princeton University Press (1971).

[9] A. Zygmund, Trigonometrical Series, 2nd ed., Cambridge University Press, Cambridge, (1959).

Received July 8, 1983.

UNIVERSITY OF WESTERN AUSTRALIA

NEDLANDS, 6009 


\section{PACIFIC JOURNAL OF MATHEMATICS EDITORS}

\author{
V. S. VARADARAJAN (Managing Editor) \\ University of California \\ Los Angeles, CA 90024 \\ Charles R. DePrima \\ California Institute of Technology \\ Pasadena, CA 91125 \\ R. FInN \\ Stanford University \\ Stanford, CA 94305
}

\section{HeRmanN FlaschKa \\ University of Arizona \\ Tucson, AZ 85721}

Ramesh A. Gangolli

University of Washington

Seattle, WA 98195

ROBION KIRBY

University of California

Berkeley, CA 94720

\section{C. MOORE}

University of California

Berkeley, CA 94720

\section{H. SAMELSON}

Stanford University

Stanford, CA 94305

HAROLD STARK

University of California, San Diego

La Jolla, CA 92093

\section{ASSOCIATE EDITORS}
R. ARENS
E. F. BECKENBACH
B. H. NeumanN
F. Wolf
K. Yoshida (1906-1982)

\section{SUPPORTING INSTITUTIONS}
CALIFORNIA INSTITUTE OF TECHNOLOGY

\author{
UNIVERSITY OF SOUTHERN CALIFORNIA \\ STANFORD UNIVERSITY \\ UNIVERSITY OF HAWAII \\ UNIVERSITY OF TOKYO \\ UNIVERSITY OF UTAH \\ WASHINGTON STATE UNIVERSITY \\ UNIVERSITY OF WASHINGTON
}
UNIVERSITY OF OREGON

\begin{abstract}
UNIVERSITY OF ARIZONA
UNIVERSITY OF BRITISH COLUMBIA

UNIVERSITY OF CALIFORNIA

MONTANA STATE UNIVERSITY

UNIVERSITY OF NEVADA, RENO

NEW MEXICO STATE UNIVERSITY

OREGON STATE UNIVERSITY
\end{abstract}

The Supporting Institutions listed above contribute to the cost of publication of this Journal, but they are not owners or publishers and have no responsibility for its content or policies.

Mathematical papers intended for publication in the Pacific Journal of Mathematics should be in typed form or offset-reproduced (not dittoed), double spaced with large margins. Please do not use built up fractions in the text of the manuscript. However, you may use them in the displayed equations. Underline Greek letters in red, German in green, and script in blue. The first paragraph must be capable of being used separately as a synopsis of the entire paper. In particular it should contain no bibliographic references. Please propose a heading for the odd numbered pages of less than 35 characters. Manuscripts, in triplicate, may be sent to any one of the editors. Please classify according to the scheme of Math. Reviews, Index to Vol. 39. Supply name and address of author to whom proofs should be sent. All other communications should be addressed to the managing editor, or Elaine Barth, University of California, Los Angeles, California 90024.

There are page-charges associated with articles appearing in the Pacific Journal of Mathematics. These charges are expected to be paid by the author's University, Government Agency or Company. If the author or authors do not have access to such Institutional support these charges are waived. Single authors will receive 50 free reprints; joint authors will receive a total of 100 free reprints. Additional copies may be obtained at cost in multiples of 50 .

The Pacific Journal of Mathematics is issued monthly as of January 1966. Regular subscription rate: $\$ 190.00$ a year (5 Vols., 10 issues). Special rate: $\$ 66.00$ a year to individual members of supporting institutions.

Subscriptions, orders for numbers issued in the last three calendar years, and changes of address should be sent to Pacific Journal of Mathematics, P.O. Box 969, Carmel Valley, CA 93924, U.S.A. Old back numbers obtainable from Kraus Periodicals Co., Route 100, Millwood, NY 10546.

The Pacific Journal of Mathematics at P.O. Box 969, Carmel Valley, CA 93924 (ISSN 0030-8730) publishes 5 volumes per year. Application to mail at Second-class postage rates is pending at Carmel Valley, California, and additional mailing offices. Postmaster: Send address changes to Pacific Journal of Mathematics, P.O. Box 969, Carmel Valley, CA 93924.

PUBLISHED BY PACIFIC JOURNAL OF MATHEMATICS, A NON-PROFIT CORPORATION

Copyright $\odot 1985$ by Pacific Journal of Mathematics 


\section{Pacific Journal of Mathematics}

Vol. 119, No. $2 \quad$ June, 1985

Mustafa Agah Akcoglu and Meira Falkowitz (Soshniak), A general local

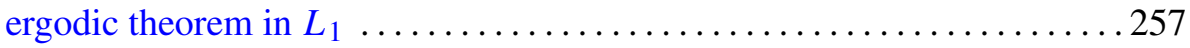

W. Wistar (William) Comfort and Lewis Chandlee Robertson,

Cardinality constraints for pseudocompact and for totally dense

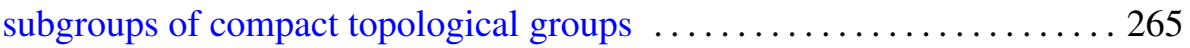

John Morse Delaurentis and Boris G. Pittel, Random permutations and

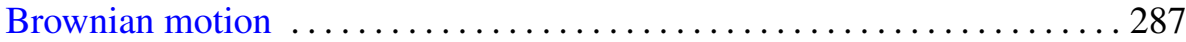

José Esteban Galé, Gel'fand theory in algebras of differentiable functions on Banach spaces ......................................... 303

Harry Gingold, On the location of zeroes of oscillatory solutions of

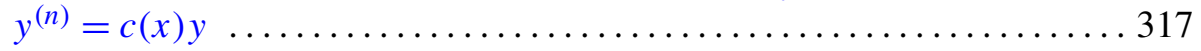

Kei Ji Izuchi, Zero sets of interpolating Blaschke products .............337

Mahesh Nerurkar, Ergodic continuous skew product actions of amenable

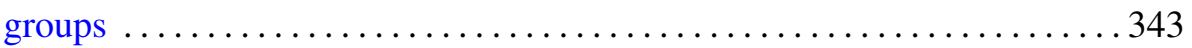

R. Owens, A maximal function characterization of a class of Hardy

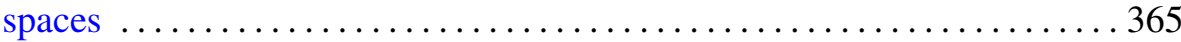

Judith Anne Packer, Point spectrum of ergodic abelian group actions and the corresponding group-measure factors $\ldots \ldots \ldots \ldots \ldots \ldots \ldots \ldots \ldots \ldots \ldots \ldots$

Judith Anne Packer, On the embedding of subalgebras corresponding to quotient actions in group-measure factors $\ldots \ldots \ldots \ldots \ldots \ldots \ldots \ldots . \ldots 4$

Iain Raeburn and Joseph L. Taylor, The bigger Brauer group and étale cohomology

David Rosen, The Diophantine equation $a x+b y=c$ in $Q(\sqrt{5})$ and other number fields

Mau-Hsiang Shih and Kok Keong Tan, Noncompact sets with convex sections

Lee Barlow Whitt, Codimension two isometric immersions between Euclidean spaces

Rodney Ian Yager, Iwasawa theory for the anticyclotomic extension 